\title{
0101. Early and severe impairment of lactate clearance in endotoxic shock is not related to liver hypoperfusion: preliminary report
}

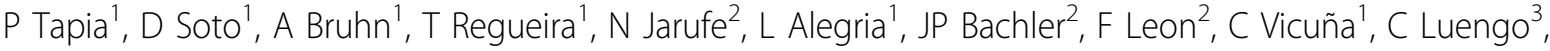 \\ G Ospina-Tascón ${ }^{4}$, J Bakker ${ }^{5}$, G Hernandez $^{1 *}$
}

From ESICM LIVES 2014

Barcelona, Spain. 27 September - 1 October 2014

\section{Introduction}

Although the prognostic value of persistent hyperlactatemia in septic shock is unequivocal, its physiological determinants are controversial. In particular, the role of impaired hepatic clearance has been considered as relevant only in severe shock with liver ischemia or advanced cirrhosis. However, very few studies have addressed this subject.

\section{Objectives}

To determine the evolution of lactate clearance [1] in an endotoxic sheep model.

\section{Methods}

This study is part of a major project exploring the influence of adrenergic stimulation and blockade over the determinants of lactate production and utilization in septic shock. Eight anesthetized sheep subjected to a multimodal hemodynamic/perfusion assessment including pulmonary artery, hepatic and portal vein catheterizations, portal/hepatic artery flow, gut tonometry, sublingual microcirculation, muscle microdialysis and hepatic mitochondrial high-resolution respirometry, were randomized to LPS or sham. LPS sheep received $5 \mathrm{mcg} / \mathrm{kg}$ bolus (E coli O127:B8 ${ }^{\circledR}$ ) and then $4 \mathrm{mcg} \cdot \mathrm{kg}$ $1 \cdot \mathrm{hr}-1$ for the rest of the experiment [2]. After $1 \mathrm{~h}$ they were volume resuscitated. Sampling and exogenous lactate clearances were performed at 4 points (fig 1 ).

'Pontificia Universidad Católica de Chile, Facultad de Medicina,

Departamento de Medicina Intensiva, Santiago, Chile

Full list of author information is available at the end of the article

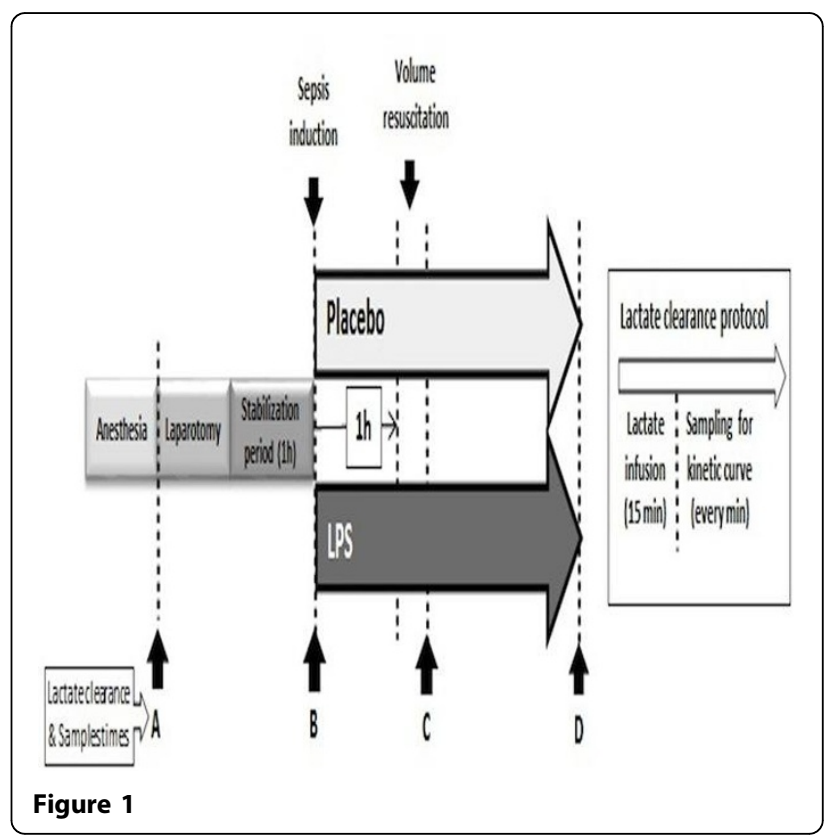

\section{Results}

LPS sheep presented an early hyperlactatemic hyperdynamic septic shock and an increased muscle lactate production compared to sham (MAP 63 vs $87 \mathrm{mmHg}$; MPAP 20 vs $10 \mathrm{mmHg}$; CO 3.7 vs $2.5 \mathrm{l} / \mathrm{min}$; arterial lactate 6.1 vs $2.6 \mathrm{mmol} / \mathrm{l}$ ). Total liver flow (865 vs 692 $\mathrm{ml} / \mathrm{min}$ ), proportion of liver flow $/ \mathrm{CO}, \mathrm{O}_{2}$ liver extraction, liver enzymes, and mitochondrial function were comparable between LPS and placebo (fig 2).

However, LPS sheep presented an early severe and persistent decrease in lactate clearance (C: 575 vs 1188 and D: 907 vs $1410 \mathrm{ml} / \mathrm{kg} / \mathrm{h}$ ), fig 3 . 


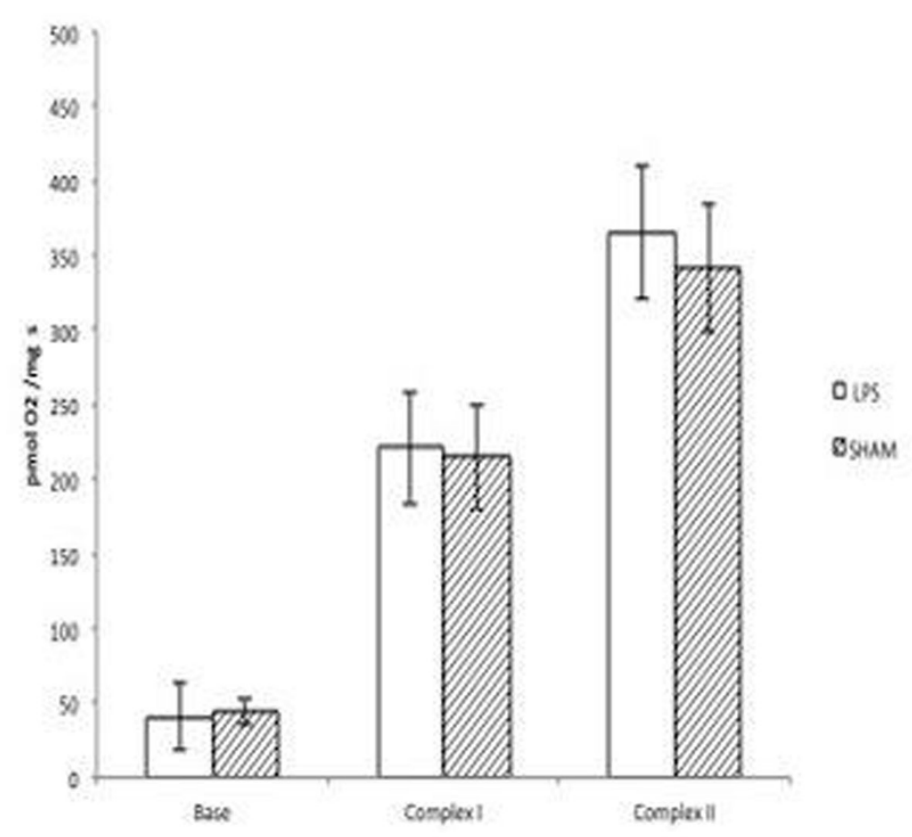

Figure 2

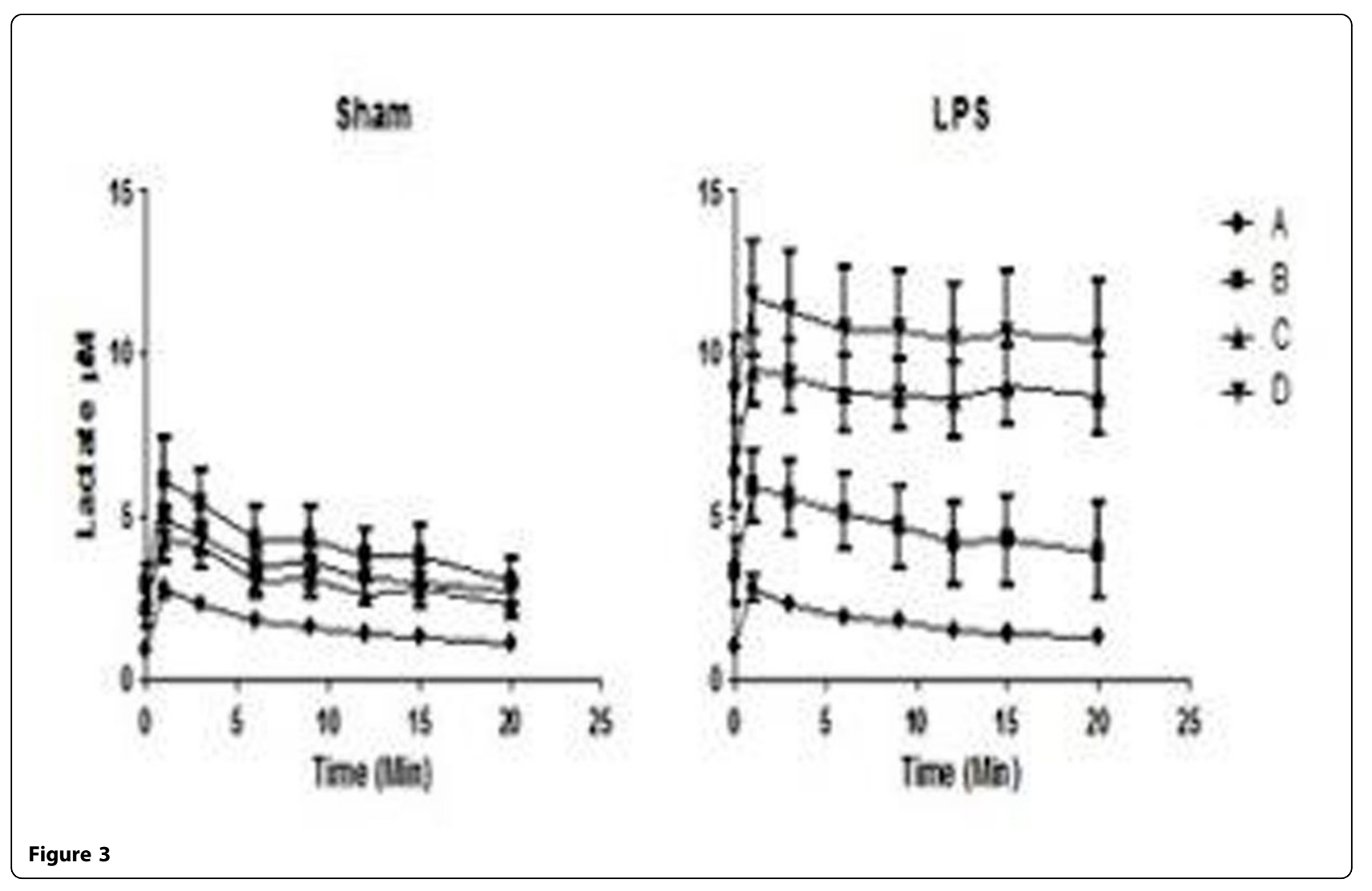




\section{Conclusions}

Hyperdynamic endotoxic shock induces an early, severe and persistent impaired lactate clearance that is not related to liver hypoerfusion, $\mathrm{O}_{2}$ extraction or mitochondrial function.

\section{Grant acknowledgment}

FONDECYT 1130200, Chile.

\section{Authors' details}

${ }^{1}$ Pontificia Universidad Católica de Chile, Facultad de Medicina,

Departamento de Medicina Intensiva, Santiago, Chile. ${ }^{2}$ Pontificia Universidad Católica de Chile, Facultad de Medicina, Departamento de Cirugía Digestiva, Santiago, Chile. ${ }^{3}$ Universidad de Chile, Hospital Clínico, Unidad de Pacientes Críticos, Santiago, Chile. ${ }^{4}$ Fundación Valle del Lili, Intensive Care Unit, Cali, Colombia. ${ }^{5}$ Erasmus MC University Medical Centre, Department of Intensive Care Adults, Rotterdam, Netherlands.

Published: 26 September 2014

\section{References}

1. Levraut J, et al: Am J Respir Crit Care Mer 1998, 157:1021-1026.

2. Dubin A, et al: Crit Care Med 2008, 36:535-542.

doi:10.1186/2197-425X-2-S1-P12

Cite this article as: Tapia et al:: 0101. Early and severe impairment of lactate clearance in endotoxic shock is not related to liver hypoperfusion: preliminary report. Intensive Care Medicine Experimental 2014 2(Suppl 1):P12.

\section{Submit your manuscript to a SpringerOpen ${ }^{\mathcal{O}}$ journal and benefit from:}

- Convenient online submission

- Rigorous peer review

- Immediate publication on acceptance

- Open access: articles freely available online

- High visibility within the field

- Retaining the copyright to your article 